\title{
O Papel dos Produtos Finais da Glicação Avançada (AGEs) no Desencadeamento das Complicações Vasculares do Diabetes
}

revisão

JÚNIA H. P. BARBOSA

Suzana L. Oliveira

Luci Tojal e Seara

Faculdade de Nutrição da Universidade Federal de Alagoas

(UFAL), Maceió, AL, Brasil.
RESUMO

Os produtos finais da glicação avançada (AGEs [do inglês, Advanced Glycation End-products]) constituem uma classe de moléculas heterogêneas formadas a partir de reações aminocarbonilo de natureza não-enzimática, que ocorrem aceleradamente no estado hiperglicêmico do diabetes. Considerados importantes mediadores patogênicos das complicações diabéticas, os AGEs são capazes de modificar, irreversivelmente, as propriedades químicas e funcionais das mais diversas estruturas biológicas. Na presente revisão, são apresentados os dados recentes da literatura que descrevem as vias de formação de AGEs, seu metabolismo, os principais mecanismos de ação dessas substâncias no desencadeamento dos processos patológicos, bem como os métodos de determinação de AGEs em amostras biológicas. Este artigo aponta, ainda, novas perspectivas de terapias anti-AGEs, a exemplo de estudos envolvendo a ação de compostos naturais dos alimentos, que podem oferecer potencial terapêutico para os portadores de diabetes ou de outras patologias associadas ao acúmulo degenerativo de AGEs. (Arq Bras Endocrinol Metab 2008; 52/6:940-950)

Descritores: Produtos finais de glicação avançada; Diabetes; Macroangiopatia; Microangiopatia

\section{ABSTRACT}

The Role of Advanced Glycation End-Products (AGEs) in the Development of Vascular Diabetic Complications.

The advanced glycation end-products (AGEs) constitute a class of heterogeneous molecules formed by amino-carbonyl reactions of a non-enzymatic nature, which occur at an accelerated rate in the hyperglycemic state of diabetes. Considered important pathogenic mediators of diabetic complications, AGEs are capable of irreversibly modifying the chemical properties and functions of diverse biological structures. In this review, recent data from literature is presented describing the pathways of AGEs formation, their metabolism, the main mechanisms of action of these substances in the triggering of pathological processes associated with diabetes, as well as methods of AGEs determination in biological samples. This text also points to new perspectives in anti-AGE therapies, an example of which is the studies involved with the action of natural compounds of food, which can represent a potential coadjuvant therapy for people with diabetes or other pathologies associated with the degenerative accumulation of AGEs. (Arq Bras Endocrinol Metab 2008; 52/6:940-950)

Recebido em 3/10/2007 Aceito em 28/3/2008
Keywords: Advanced glycation endproducts; Diabetes; Macroangiopathy; Microangiopathy 


\section{INTRODUÇÃO}

A S COMPLICAÇÕES VASCULARES do diabetes melito são a principal causa de morbimortalidade nos países desenvolvidos e constituem preocupação crescente para as autoridades de saúde em todo o mundo (1). Entre as teorias que explicam como a hiperglicemia crônica conduz aos danos celulares e teciduais observados nessa doença, a formação dos produtos de glicação avançada, também chamados AGEs (do inglês, Advanced Glycated End-Products), é considerada uma das mais importantes $(2,3)$.

Os efeitos patológicos dos AGEs estão relacionados à capacidade destes compostos de modificar as propriedades químicas e funcionais das mais diversas estruturas biológicas. Por meio da geração de radicais livres, da formação de ligações cruzadas com proteínas ou de interações com receptores celulares, os AGEs promovem, respectivamente, estresse oxidativo, alterações morfofuncionais e aumento da expressão de mediadores inflamatórios. A contribuição dos AGEs para o desenvolvimento e a progressão das complicações diabéticas encontra-se bem evidenciada na literatura (4-6).

A atual pandemia de diabetes tem estimulado pesquisas sobre sua intrincada fisiopatologia, a fim de que haja melhora na detecção, na prevenção e no tratamento das complicações associadas. Nesse sentido, este trabalho tem como objetivo descrever o papel dos AGEs como importantes mediadores das diversas complicações diabéticas. Para tanto, serão tratados as vias de formação de AGEs, seu metabolismo, os principais mecanismos de ação dessas substâncias no desencadeamento dos processos patológicos, bem como os métodos de determinação de AGEs em amostras biológicas. Este artigo aponta, ainda, novas perspectivas de terapias anti-AGEs, a exemplo de estudos envolvendo a ação de compostos naturais dos alimentos.

\section{PRODUTOS FINAIS DA GLICAÇÃO AVANÇADA (AGEs)}

Os produtos finais da glicação avançada (AGEs) constituem grande variedade de substâncias formadas a partir de interações amino carbonilo, de natureza não-enzimática, entre açúcares redutores ou lipídeos oxidados e proteínas, aminofosfolipídeos ou ácidos nucléicos (7). Embora parte dos mecanismos que levam à formação dos AGEs permaneça ainda desconhecida, a química destas complexas reações pode ser esquematizada como mostra a Figura 1.

A via clássica da reação de Maillard, também denominada glicação, inicia-se com a formação de base de Schiff instável, gerada pela condensação de grupamento carbonila de açúcar redutor, como a glicose, com um grupamento amina, oriundo, por exemplo, do aminoácido lisina, composto especialmente suscetível à reação. Na seqüência, a base de Schiff sofre rearranjos, tornando essa estrutura mais estável, o produto de Amadori, modernamente conhecido como early Maillard reaction products (early MRPs) ou produtos iniciais da reação de Maillard. A hemoglobina glicosilada e a frutosamina são conhecidos exemplos de produtos de Amadori. Os produtos de Amadori gerados possuem grupos carbonilas reativos, que se condensam com grupos aminas primários acessíveis, dando origem aos produtos avançados da reação de Maillard - AGEs (6,7).

Os mecanismos alternativos de formação de AGEs incluem a chamada via do "estresse carbonílico", na qual a oxidação de lipídeos ou de açúcares gera compostos dicarbonílicos intermediários altamente reativos (8). A glicólise e a autoxidação de glicose, por exemplo, produzem metilglioxal e glioxal, que interagem com aminoácidos para formar AGEs. Estes compostos dicarbonílicos chegam a ser 20 mil vezes mais reativos do que a glicose e são os principais intermediários da formação de AGEs (9). Os AGEs formados a partir da oxidação de açúcares ou de lipídeos podem também ser denominados, respectivamente, produtos da glicoxidação ou da lipoxidação avançada. Deve-se ressaltar que, durante algumas das reações que levam à formação de AGEs, espécies reativas do oxigênio ( $\mathrm{ROS}$ ) são geradas e concorrem paralelamente com o estresse oxidativo e com os danos estruturais e funcionais às macromoléculas $(10,11)$.

A formação de AGEs in vivo pode, adicionalmente, envolver neutrófilos, monócitos e macrófagos, os quais, após estímulo inflamatório, produzem mieloperoxidase e a enzima NADPH oxidase, que induzem a formação de AGEs por meio da oxidação de aminoácidos $(8)$.

Em virtude da complexidade e da heterogeneidade das reações que podem ocorrer, apenas poucos AGEs foram claramente identificados e podem ser quantificados em estudos laboratoriais. A carboximetilisina (CML), a pirralina e a pentosidina são exemplos de AGEs bem caracterizados e amplamente estudados $(5,12)$. 


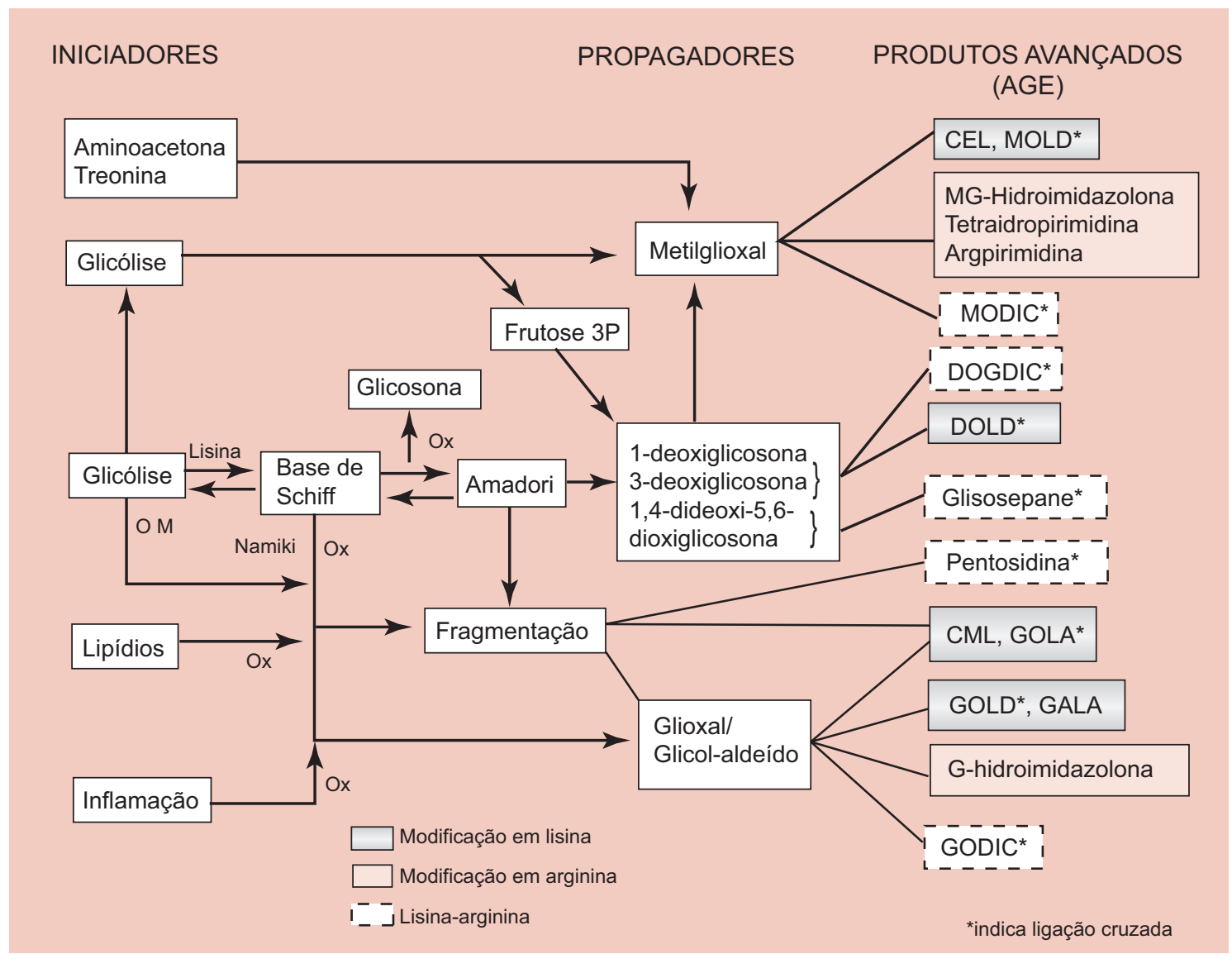

CML: carboximetilisina; carboxietilisina (CEL); DOGDIC: ligação cruzada imidazolina derivada de 3-deoxiglicosona; DOLD: dímero de lisina e 3-deoxiglicosona; GOLA glioxal lisina amida; GOLD: dímero de glioxal-lisina, GALA: ácido glioxilico lisina amida; GODIC: ligação cruzada imidazolina derivada de glioxal; MODIC: ligação cruzada imidazolina derivada de metil glioxal; MOLD: dímero de metilglioxal-lisina (adaptado 7).

Figura 1. Rotas de formação e produtos finais de glicação avançada (AGE).

\section{METABOLISMO DE AGEs}

O pool endógeno de AGEs reflete basicamente o balanço cinético de dois processos opostos: a formação endógena e a absorção de AGEs exógenos, de um lado, e a degradação e a eliminação de AGEs por sistemas especializados, de outro (4).

A formação de AGEs ocorre vagarosamente sob condições fisiológicas, e afeta predominantemente moléculas de meia-vida longa, como o colágeno, exercendo importante função no processo de envelhecimento (13). No entanto, sob condições de hiperglicemia ou estresse oxidativo, a geração de AGEs aumenta intensamente $(10,14)$. Os portadores de diabetes apresentam concentrações séricas de AGEs significativamente mais altas que os indivíduos não-diabéticos (15). A mensuração da hemoglobina glicosilada $\left(\mathrm{HbA}_{1 \mathrm{C}}\right)$, variante de hemoglobina que carrega um produto da Amadori em sua cadeia $\beta$, reflete a ocorrência de hiperglicemias nos últimos três meses e, indiretamente, de glicação avançada. A HbA 1 é importante marcador biomolecular e a sua determinação é considerada, até o presente, o indicador mais confiável de progressão do diabetes (16).

Acreditava-se, originalmente, que os AGEs formavam-se, em primeiro lugar, a partir de reações não enzimáticas entre glicose e proteínas extracelulares. Porém, por causa da maior reatividade dos precursores dicarbonílicos derivados da glicose gerados intracelularmente (glioxal, metilglioxal e 3-deoxiglicosona), a alta concentração de glicose intracelular é considerada atualmente o evento iniciador primário da formação de AGEs intra e extracelulares $(2,17)$.

A formação de AGEs é predominantemente endógena, mas esses produtos podem ser introduzidos no 
organismo por fontes exógenas, como o fumo e a dieta $(3,18)$.

A dieta é considerada a principal fonte exógena de AGEs e pode exercer importante influência no desenvolvimento de diversos quadros patológicos, especialmente do diabetes (19). Sabe-se que, aproximadamente, $10 \%$ dos AGEs ingeridos com a dieta são absorvidos, embora os mecanismos referentes a essa absorção não estejam totalmente esclarecidos. Da fração absorvida, cerca de dois terços são retidos no organismo e apenas um terço é excretado pela urina, dentro de 48 horas, por indivíduos com função renal normal $(8,20)$. A formação de AGEs nos alimentos é potencializada por métodos de preparo que utilizam altas temperaturas e baixa umidade (fritar, assar ou grelhar), sendo os alimentos ricos em lipídeos os principais contribuintes do conteúdo dietético de AGEs (2l). Há evidências de que os AGEs dietéticos se somam ao pool de AGEs endógenos, favorecendo o surgimento e a progressão das diversas complicações do diabetes $(3,19)$.

O fumo é também considerado importante fonte exógena de AGEs (3). Durante a combustão do tabaco, espécies reativas de AGEs são volatilizadas, absorvidas pelos pulmões e podem interagir com proteínas séricas (22). Isso se reflete no fato de que as concentrações séricas de AGEs e de AGE-apoproteína B em fumantes se apresentam significativamente mais altas que em não-fumantes (23).

O organismo possui mecanismos de defesa contra o acúmulo degenerativo de AGEs. Os sistemas enzimáticos capazes de influenciar o pool endógeno de AGEs incluem a oxaldeído redutase e a aldose redutase, eficientes na detoxificação de intermediários dicarbonílicos reativos. Os sistemas enzimáticos glioxilase I e II, a frutosamina-3-cinase e a frutosamina oxidase (amadoriase) são também responsáveis por interromper reações de glicação em diferentes estágios (24). Porém, sob condições de excesso de AGEs, como no diabetes, hiperlipidemia, insuficiência renal e em indivíduos que consomem dieta com alto conteúdo em AGEs, esses sistemas podem ser superados (25).

A remoção dos AGEs formados nos componentes teciduais é realizada pela proteólise extracelular ou pelas células scavenger, como os macrófagos, que endocitam AGEs via receptores e, após a degradação intracelular, liberam na circulação AGE-peptídeos solúveis e de baixo peso molecular, para serem excretados com a urina (6). Entre esses AGE-peptídeos, também denominados "segunda geração de AGEs", pode haver in- termediários altamente reativos, mas seus efeitos são limitados pela excreção renal (26). Assim, a eficiência dos sistemas de remoção de AGEs depende, em última instância, da eficiência do clearance renal. A disfunção renal que ocorre em pacientes portadores de nefropatia, por exemplo, resulta na falha da remoção dos AGEs circulantes e contribui consideravelmente para as altas concentrações de AGEs séricos e teciduais encontradas nesses pacientes (27).

Adicionalmente, a lisozima, proteína com reconhecida propriedade antimicrobial, possui alta afinidade por AGEs e é capaz de auxiliar na remoção desses compostos (8). Em estudos in vivo e in vitro, a lisozima demonstrou propriedades protetoras importantes, como a melhora da excreção renal e a diminuição das concentrações séricas de AGEs, além da supressão da ativação celular adversa mediada por AGEs (28). Todavia, os mecanismos envolvidos nesses achados estão ainda sob investigação (22).

Associados a esses processos de formação/absorção e degradação/eliminação, os fatores genéticos podem influenciar o metabolismo de AGEs nos indivíduos e, conseqüentemente, a predisposição para o desenvolvimento de patologias associadas a estes compostos, como o diabetes, a aterosclerose, a artrite, a osteoporose e a doença de Alzheimer $(18,29)$. Como exemplo, o polimorfismo do RAGE, um dos reconhecidos receptores de AGEs, foi associado a um leve efeito protetor quanto ao desenvolvimento de nefropatia em portadores de diabetes tipo 1 , sugerindo importante impacto da expressão gênica no surgimento de complicações vasculares do diabetes (30).

\section{MECANISMOS DE AÇÃO DOS AGEs}

Os AGEs podem danificar as células por três mecanismos básicos. O primeiro é a modificação de estruturas intracelulares, incluindo aquelas envolvidas com a transcrição gênica. O segundo mecanismo é a interação de AGEs com proteínas da matriz extracelular modificando a sinalização entre as moléculas da matriz e a célula, gerando disfunção. $\mathrm{O}$ terceiro mecanismo se refere à modificação de proteínas ou lipídeos sangüíneos; as proteínas e os lipídeos circulantes modificados por AGEs podem, então, ligar-se a receptores específicos, causando a produção de citocinas inflamatórias e fatores de crescimento, que, por sua vez, contribuem para a patologia vascular do diabetes $(2,6,17)$. 
Os AGEs formados intracelularmente podem alterar propriedades celulares importantes para a homeostase vascular. A modificação por AGEs do bFGF (basic fibroblast growth factor), por exemplo, reduz em $70 \%$ a atividade mitogênica do citosol das células endoteliais (31). Mais relevantes são as alterações genômicas que podem ocorrer após a interação entre AGEs e nucleotídeos, histonas ou proteínas envolvidas na transcrição do DNA celular (17).

As proteínas de meia-vida longa que constituem a matriz extracelular e as membranas basais vasculares são também suscetíveis à formação e ao acúmulo de AGEs. Além de reduzir a solubilidade e a digestibilidade enzimática, a formação de AGEs compromete a conformação geométrica dessas proteínas, causando anormalidades estruturais e funcionais permanentes. Há evidências de que a formação de ligações cruzadas entre AGEs e o colágeno tipo I ou elastina conduza ao aumento na área da matriz extracelular, resultando aumento da rigidez da vasculatura. A formação de AGEs na laminina compromete a reunião de seus polímeros, diminuindo, assim, sua interação com o colágeno tipo IV e com o proteoglicano de heparam sulfato $(17,31)$.

Os AGEs não interferem apenas nas interações entre os componentes da matriz extracelular, mas também nas interações entre as células e a matriz. Um bom exemplo dessa interferência consiste da diminuição da adesão das células endoteliais por causa das alterações causadas por AGEs nos domínios de ligações celulares do colágeno tipo IV (2).

Entre os mecanismos pelos quais os AGEs podem contribuir para o desenvolvimento e a progressão das complicações vasculares do diabetes, a interação desses compostos com receptores presentes na superfície de diversos tipos celulares merece papel de destaque. Desde as primeiras pesquisas sobre AGEs in vivo, especulou-se sobre a possível existência de sistema de receptores para AGEs, responsável pela remoção desses produtos nos tecidos, limitando seus efeitos deletérios. Demonstrou-se, originalmente, que as proteínas modificadas por AGEs eram reconhecidas por receptores específicos que foram associados aos sistemas de receptores scavenger. Hoje, sabe-se da existência de um complexo sistema de reconhecimento de AGEs, que exerce importante função na patologia associada ao diabetes (22).

Entre uma variedade de receptores de AGEs ou proteínas ligantes de AGEs que têm sido descritos na literatura, o receptor RAGE é provavelmente a molécula mais bem caracterizada $(32,33)$. O RAGE pertence à superfamília das imunoglobulinas de superfície celular, estando seu gene localizado no cromossomo 6 no complexo principal de histocompatibilidade entre os genes para as classes II e III. As regiões para o fator nuclear- $\kappa B$ (NF-кB) e para a interleucina-6 (IL-6) estão localizadas no gene promotor do RAGE, controlando a expressão desse receptor e associando o RAGE às respostas inflamatórias. O RAGE é minimamente expresso nos tecidos e na vasculatura sob condições fisiológicas. No entanto, sua expressão está aumentada em macrófagos, monócitos, células musculares lisas, células endoteliais e astrócitos sob condições de excesso de AGEs, um exemplo de feedback positivo $(31,32)$.

A interação AGE-RAGE nas células endoteliais ativa

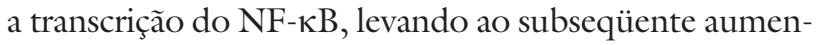
to da expressão de seus genes-alvo, como endotelina- 1 , vascular cell adhesion molecule-1 (VCAM-1), selectina E, fator tecidual, trombomodulina, VEGF (vascular endothelial growth factor) e de citocinas pró-inflamatórias que incluem a interleucina- $1 \alpha$ (IL-l $\alpha$ ), (IL-6) e o fator de necrose tumoral- $\alpha$ (TNF- $\alpha$ ), além do próprio RAGE. O bloqueio do RAGE, por sua vez, inibe a ativação do NF- $\kappa \mathrm{B}(31)$.

Há evidências que comprovam o envolvimento do RAGE no desenvolvimento das macro e microangiopatias diabéticas. Shoji e cols. (34) demonstraram, por exemplo, que a presença do RAGE é essencial para o comprometimento da resposta angiogênica no diabetes e que o bloqueio funcional desse receptor é capaz de restaurar a resposta angiogênica suprimida. Em um outro estudo, a inativação do gene para o RAGE em um modelo animal de nefropatia diabética resultou supressão significativa das modificações renais características e evidenciou que o grau da injúria renal era proporcional à dosagem de genes para o RAGE (35).

Outros receptores, como os denominados AGE-Rl, AGE-R2 e AGE-R3, e os receptores scavenger de macrófagos classe A tipos I e II, também são capazes de reconhecer e ligar-se a AGEs, mas não demonstraram qualquer atividade de transdução de sinal após a interação com AGEs. Ao contrário, eles são associados ao clearance e à detoxificação desses compostos. $\mathrm{O}$ denominado CD36, receptor scavenger da classe B, similarmente reconhece AGEs e está envolvido no clearance desses compostos da circulação, mas exerce importante função na indução do estresse oxidativo na célula. Os AGEs também são reconhecidos pelos receptores scavenger classe $\mathrm{E}$, lectin-like oxidized LDL receptor-1 (LOX-1), fasciclina, epidermal growth factor-like (EGF), 
laminin-type epidermal growth factor-like (LE), link domain-containing scavenger receptor-1 e 2 (FEEL-1 e FEEL-2) (31).

A modulação das diferentes atividades exercidas pelos receptores de AGEs constitui campo de grande interesse para os investigadores e tem sido associada a diversos fatores, como concentração de glicose, insulina, AGEs e espécies reativas de oxigênio (ROS) em vários tipos de células. As medidas freqüentemente utilizadas em estudos para avaliar a modulação dos receptores de AGEs centram-se nos parâmetros de ativação celular (22).

A caracterização de moléculas de receptores de AGEs, de suas organizações genômicas, bem como de seus polimorfismos genéticos, têm sido elucidados e poderão contribuir para o desenvolvimento de novas terapias anti-AGEs (22).

\section{AGES E AS COMPLICAÇÕES DIABÉTICAS}

Os AGEs são considerados importantes mediadores patogênicos das complicações do diabetes, convencionalmente classificadas em micro ou macroangiopatias. A microangiopatia diabética é termo geral utilizado para denominar as modificações funcionais dos leitos microvasculares, nos quais o endotélio e as células associadas são progressivamente danificados pela hiperglicemia, resultando oclusão capilar, isquemia e falência de órgãos. Já o termo macroangiopatia diabética refere-se às complicações cardiovasculares, que constituem a principal causa de morbimortalidade em pacientes com diabetes melito. Ambos os tipos de diabetes (l 1 e 2 ) representam fator de risco independente e poderoso para doença arterial coronariana, infarto e doença arterial periférica $(25,36)$.

O envolvimento dos AGEs na patogenia das micro e macroangiopatias diabéticas é fato bem estabelecido na literatura, embora alguns dos mecanismos que os envolvem e que conduzem aos danos celulares e teciduais característicos estejam, ainda, sob investigação (22).

\section{AGEs e a microangiopatia diabética}

No diabetes, as células especialmente afetadas pela hiperglicemia são as células endoteliais dos capilares da retina, as células mesangiais do glomérulo renal, os neurônios e as células de Schwann nos nervos periféricos. Isso se deve à incapacidade desses tipos celulares regularem o transporte de glicose para o meio interno, ficando suscetíveis às altas concentrações de glicose durante os estados hiperglicêmicos. Essas altas concentrações intracelulares de glicose, por sua vez, estimularão as vias bioquímicas responsáveis pelas alterações fisiopatológicas do diabetes, quais sejam, via do poliol, via da hexosamina, via dos AGEs e via da proteína quinase C-, entre as quais destaca-se a via de formação dos AGEs $(2,17)$.

\section{Retinopatia diabética}

Apesar de anos de investigações clínicas e laboratoriais, a retinopatia diabética permanece a principal causa da deterioração da visão e da cegueira entre os adultos em idade produtiva, sendo clinicamente diagnosticada a partir do desenvolvimento de sinais oftalmoscópicos, como microaneurismas, hemorragias e manchas características que afetem a retina (37). A natureza multifatorial das várias vias implicadas nessa patologia constitui o obstáculo para o desenvolvimento de estratégias terapêuticas eficientes, embora se saiba do importante envolvimento dos AGEs no surgimento e progressão da retinopatia diabética $(38)$.

Os AGEs podem ser detectados nos vasos sangüíneos da retina e acredita-se que sejam responsáveis pelo aumento da permeabilidade das células endoteliais e que contribuam para a oclusão vascular (39). Os pericitos expressam receptores RAGE que, ao interagirem com seus ligantes AGEs, desencadeiam o aumento na transdução de sinais celulares e causam um dos fenômenos característicos da retinopatia diabética: a perda dos pericitos. Nas células endoteliais, os AGEs-RAGE causam a superprodução do fator de crescimento das células do endotélio vascular (VEGF), que, por sua vez, estimula a angiogênese e a neovascularização, ambas também envolvidas na patogênese da retinopatia (5).

\section{Nefropatia diabética}

A nefropatia diabética é a principal causa de doença renal em estágio terminal nos países desenvolvidos (40). A nefropatia é caracterizada pelo desenvolvimento de proteinúria, aumento da pressão sangüínea sistêmica e declínio da função renal (41).

Embora os eventos moleculares precisos que levam à nefropatia diabética estejam ainda sendo investigados, sabe-se que a membrana basal glomerular, as células mesangiais, os podócitos e as células tubulares renais acumulam altos níveis de AGEs. Os AGEs (via RAGE) aumentam a liberação do fator de crescimento $\beta$ (TGF- $\beta$ ), que estimula a síntese de componentes da 
matriz do colágeno, fenômeno este que deve contribuir, ao menos parcialmente, para o espessamento da membrana basal, característico da nefropatia diabética. $\mathrm{O}$ acúmulo de AGEs no colágeno da membrana basal, aliado à capacidade desses compostos de se ligarem a proteínas plasmáticas, pode também contribuir para o espessamento da membrana basal, alterações na filtração e, finalmente, para a perda da função glomerular. A redução da filtração é, em parte, por causa da compressão dos capilares e da diminuição da superfície de área onde ocorre a filtração, por causa da expansão do leito mesangial. As células mesangiais de cultura possuem receptores para AGEs e respondem aos AGEs com o aumento da síntese de proteínas da matriz e de colágeno tipo IV (22).

\section{Neuropatia diabética}

Embora seja alta a incidência de neuropatia periférica em pessoas diabéticas, é difícil estabelecer a prevalência deste distúrbio em virtude da diversidade das suas manifestações clínicas. A neuropatia diabética é caracterizada pela desmielinização segmentar e degeneração axonal dos neurônios periféricos, acompanhada de anormalidades funcionais, como a redução da condução nervosa e do fluxo sangüíneo. Pode apresentar-se clinicamente pela diminuição das percepções de vibrações, dor e temperatura, especialmente nas extremidades inferiores. Além disso, na neuropatia do sistema nervoso autônomo há defeitos nas respostas vasomotoras, diminuição das respostas cardíacas, distúrbio da motilidade do trato gastrintestinal, incapacidade de esvaziar a bexiga e disfunção sexual (42).

Existe aumento da glicação da mielina nos diabéticos. A mielina glicada é suscetível à fagocitose por macrófagos, que também podem ser estimulados a secretar proteases, contribuindo para a desmielinização do nervo na neuropatia diabética. Adicionalmente, os AGEs na mielina podem se ligar a proteínas plasmáticas, como a IgG e a IgM e estimular reações imunes, que potencializem a desmielinização neuronal. Com base em estudos experimentais, evidenciou-se que a disfunção neuronal está estreitamente associada com a ativação do NF- $\kappa B$ e com a expressão de citocinas pró-inflamatórias, como a IL- 6 e o TNF- $\alpha$. Considerando-se que a

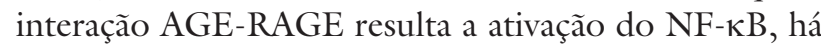
a hipótese de que os AGEs possam contribuir significativamente para a disfunção neuronal no diabetes (5). Os nervos de pacientes diabéticos apresentam reatividade imunológica aumentada para CML e RAGE, consis- tente com a hipótese da via AGE-RAGE-NF- $\kappa$ B estar supra-regulada nos nervos periféricos dos diabéticos (43). Os AGEs podem também estar envolvidos no processo de espessamento dos vasos sangüíneos que suprem o nervo, ocasionando isquemia vascular. A isquemia vascular é supostamente fator importante no desenvolvimento dessas alterações neurais (5).

\section{Comprometimento da cicatrização de feridas no diabetes}

A cicatrização normal de feridas constitui seqüência complexa e coordenada de eventos, que envolvem migração de células específicas para o local, inflamação, proliferação de diferentes tipos de células, angiogênese, formação de componentes da matriz, remodelamento e, finalmente, fechamento. O comprometimento da cicatrização de feridas no indivíduo diabético é a principal causa de grande número de amputações e, conseqüentemente, do aumento da morbimortalidade (44).

A resposta inflamatória que sucede à lesão é importante para a rápida cicatrização da ferida. No diabetes, há inicialmente retardo no afluxo de células inflamatórias para o local do ferimento, mas, quando estas células se estabelecem, ocorre então estado de inflamação crônica, prevenindo a deposição de componentes da matriz, o remodelamento e, finalmente, o fechamento da ferida. A resposta inflamatória sustentada é acompanhada pela interação AGEs-RAGE, que estimula a liberação de moléculas pró-inflamatórias, como o TNF- $\alpha$ e as metaloproteinases (MMPs) destruidoras da matriz, as quais limitam o fechamento da ferida. Além disso, a interação AGE-RAGE nos fibroblastos pode causar a redução da deposição necessária do colágeno, comprometendo ainda mais o processo normal de cicatrização (5).

\section{AGEs e a macroangiopatia diabética}

Em contraste com as células envolvidas na doença microvascular do diabetes, nas células do endotélio arterial, capazes de regular a concentração interna de glicose, ocorre o aumento do fluxo de ácidos graxos livres provenientes dos adipócitos por causa da resistência à ação da insulina ou a falta desse hormônio. $\mathrm{O}$ conseqüente aumento da oxidação desses ácidos graxos pela mitocôndria leva à superprodução de espécies reativas de oxigênio (ROS) pela cadeia de transporte de elétrons mitocondrial, o que ativa as mesmas vias bioquímicas responsáveis pelas alterações fisiopatológicas do diabetes: via do poliol, via da he- 
xosamina, via da proteína quinase $\mathrm{C}$ e via de formação dos $\operatorname{AGEs}(2,17)$.

Os AGEs podem ser altamente deletérios à integridade e à função dos vasos sangüíneos de diversas maneiras. No interior da parede dos vasos, os AGEs formam ligações cruzadas intra e intermoleculares em proteínas da matriz, "aprisionam" proteínas plasmáticas, causam enrijecimento da vasculatura, extinguem a atividade do óxido nítrico $(\mathrm{NO})$ e da prostaciclina $\left(\mathrm{PGI}_{2}\right)$, além de interagirem com receptores para modular grande número de propriedades celulares. Os AGEs não apenas diminuem os níveis desses dois importantes fatores relaxantes derivados do endotélio, mas também induzem a produção do potente vasoconstritor endotelina-1 pelas células endoteliais por meio da ativação do NF- $\kappa$ B. Nas proteínas de baixa densidade plasmáticas (LDL), os AGEs iniciam reações oxidativas que promovem a formação da LDL oxidada. A interação dos AGEs com células que se acumulam nas placas arterioscleróticas, como os fagócitos mononucleares e as células musculares lisas, gera estímulos que intensificam a resposta inflamatória. Adicionalmente, a interação dos AGEs com componentes da parede dos vasos aumenta a permeabilidade vascular, a expressão da atividade pró-coagulante e a geração de ROS, resultando expressão aumentada de moléculas de adesão leucocitárias endoteliais. Os AGEs modulam os estágios iniciais da arterogênese, desencadeando processo inflamatório-proliferativo e contribuindo para a propagação da inflamação e para a perturbação vascular na doença já estabelecida $(6,31,36)$.

\section{AGES COMO MARCADORES DO DIABETES}

A estrutura de número crescente de AGEs tem sido elucidada e acredita-se que a mensuração desses produtos no soro ou nos tecidos dos portadores de diabetes poderá ser útil para avaliar o risco de progressão da doença. Diversos estudos têm utilizado o colágeno da pele ou proteínas plasmáticas para investigar correlações dos diferentes AGEs com a gravidade das complicações diabéticas (45). Os produtos de glicoxidação CML e pentosidina foram associados à nefropatia e à retinopatia (46). A neuropatia está associada a quase todos os marcadores avaliados, sugerindo que o nervo seja mais suscetível ao estresse carbonílico total que a vias específicas de formação de AGEs. No entanto, existe ainda considerável incerteza em relação à contribuição efetiva de cada AGE para as alterações celulares e teciduais observadas no diabetes. Incerteza adicional reside nas limitações dos diferentes ensaios utilizados para quantificar os AGEs, entre elas a falta de propriedade que caracterize todo o grupo (45).

A fluorescência, sob comprimentos de onda entre 370 e $440 \mathrm{~nm}$, é utilizada para mensurar AGEs, mas a natureza da fluorescência pode ser altamente heterogênea e não relacionada aos produtos da glicação. A pentosidina pode ser quantificada pela cromatografia líquida de alta performance (HPLC), porém a hidrólise ácida utilizada nessa técnica pode gerar artefatos que interfiram no resultado. A CML é mais precisamente mensurada pela cromatografia gasosa associada à espectrometria de massa $(\mathrm{GC} / \mathrm{MS})$ ou pela cromatografia líquida associada à espectrometria de massa (LC/MS), mas esses métodos se tornam inconvenientes para grande número de amostras. Testes imunoreativos que utilizam anticorpos mono ou policlonais especificamente desenvolvidos também são utilizados em investigações sobre AGEs, mas apresentam baixa sensibilidade (45).

Uma inovação nos experimentos clínicos diz respeito à utilização de leitor de autofluorescência (AFR [do inglês autofluorescence reader]), que quantifica o acúmulo de AGEs na pele de maneira não-invasiva $\mathrm{e}$ poderá se tornar ferramenta útil para a avaliação do risco de complicações crônicas do diabetes. O AFR foi recentemente validado e estudos preliminares realizados com pacientes diabéticos confirmaram as correlações entre idade, controle glicêmico, função renal e autofluorescência da pele (47-49).

\section{PERSPECTIVAS}

Os AGEs contribuem de maneira clara e relevante para o surgimento e a progressão das complicações do diabetes, representando alvo promissor para intervenções terapêuticas. Uma variedade de agentes que apresentam propriedades anti-AGE está sendo atualmente investigada e pode atuar de diversas maneiras, incluindo a diminuição da absorção de AGEs, a inibição da formação de produtos de Amadori, a prevenção da progressão dos produtos de Amadori a AGEs, a diminuição do estresse oxidativo, a ligação e detoxificação de intermediários dicarbonílicos e a interrupção de vias bioquímicas capazes de causar impacto nos níveis de AGEs. Entre esses agentes estão medicamentos, suplementos e terapias dietéticas. 
Os medicamentos investigados incluem aminoguanidina, aspirina, OBP 9195, ALT-946, ALT-711, metformina e bloqueadores de receptor da angiotensina-II e, embora nenhum deles tenha sido ainda aprovado para indicação específica anti-AGE, alguns já se encontram em fases pré-clínica e clínica de testes $(7,8)$. A aminoguanidina, composto de hidrazina que se encontra em fase de teste em humanos, previne a formação de AGEs ao reagir com produtos iniciais da glicação e demonstrou ser eficaz na melhora da proteinúria, da elasticidade dos vasos e na prevenção da retinopatia diabética. Porém, alguns efeitos colaterais associados ao seu uso crônico, que incluem a maior incidência de glomerulonefrite e a deficiência da vitamina B6, têm exigido o esforço dos pesquisadores para estabelecer dosagem segura para sua utilização terapêutica em portadores de diabetes $(8,31,50)$.

Em outra linha de investigações, a restrição dietética de AGEs tem emergido como estratégia efetiva e viável por demonstrar efeitos benéficos em relação a arteriosclerose (51), uremia (52), proliferação da íntima após angioplastia (53), sensibilidade à insulina $(54,55)$, cicatrização de feridas $(56)$ e prevenção do diabetes tipo 1 (57) com a simples modificação dos métodos de preparo dos alimentos. Em um estudo envolvendo seres humanos, a restrição do consumo de alimentos fritos, grelhados ou assados, por períodos que variaram de duas a seis semanas, foi responsável pela diminuição significativa dos níveis séricos de AGEs e de mediadores inflamatórios envolvidos na angiopatia diabética (58).

Outro aspecto que tem atraído o interesse de pesquisadores é a presença de compostos que apresentem propriedades de antiglicação e/ou antioxidantes nos alimentos, que possam oferecer potencial terapêutico para os portadores de diabetes ou de outras patologias associadas ao acúmulo degenerativo de AGEs. Embora nenhum alimento ou componente alimentar seja ainda especificamente indicado como redutor de AGEs in vivo, algumas substâncias demonstraram efeitos antiAGE importantes e merecem investigações adicionais, como piridoxamina $(7,59)$, alilcisteína (componente do extrato de alho) (60), compostos fenólicos (61-64), vitaminas C e E $(65)$, tiamina $(66,67)$, taurina $(68)$ e carnosina (69).

Os recentes avanços no conhecimento da formação, identificação e ação dos AGEs têm criado novas possibilidades de diagnóstico, monitoramento, prevenção e tratamento das patologias associadas ao diabetes.
No entanto, muitos estudos são ainda necessários para a elucidação de questões estratégicas relativas à repercussão desses compostos nesse contexto, a fim de estabelecer intervenções seguras que melhorem a qualidade de vida dos milhões de portadores dessa enfermidade.

\section{REFERÊNCIAS}

1. Zimmet P, Alberti KG, Shaw J. Global and societal implications of the diabetes epidemic. Nature. 2001;414:782-7.

2. Brownlee M. Biochemistry and molecular cell biology of diabetic complications. Nature. 2001;414:813-20.

3. Peppa M, Uribarri J, Vlassara H. Glucose, advanced glycation end products, and diabetes complications: what is new and what works. Clin Diabetes. 2003;21(4):186-7.

4. Jakus V, Rietbrock N. Advanced glycation end products and the progress of diabetic vascular complications. Physiol Res. 2004;53(2):131-42.

5. Ahmed N. Advanced glycation endproducts-role in pathology of diabetic complications. Diabetes Res Clin Pract. 2005;67(1):3-21.

6. Bierhaus A, Hofman MA, Ziegler R, Nauroth PP. AGEs and their interaction with AGE-receptors in vascular disease and diabetes mellitus. I. The AGE concept. Cardiovasc Res. 1998;37(3):586-600.

7. Monnier VM. Intervention against the Maillard reaction in vivo. Arch Biochem Biophys. 2003;419(1):1-15.

8. Huebschmann AG, Regensteiner JG, Vlassara $H$, Reusch JE. Diabetes and glicoxidation end products. Diabetes Care. 2006;29(6):1420-32.

9. Meade SJ, Miller AG, Gerrard JA. The role of dicarbonyl compounds in non-enzimatic crosslinking: a structure-activity study. Bioorg Med Chem. 2003;11(6):853-62.

10. Jay D, Hitomi H, Griendling KK. Oxidative stress and diabetic cardiovascular complications. Free Radic Biol Med. 2006; 40(2):183-92.

11. Hidalgo FJ, Zamora R. Interplay between the maillard reaction and lipid peroxidation in biochemical systems. Ann N Y Acad Sci. 2005;1043:284-9.

12. Henle T. AGEs in food: do they play a role in uremia? Kidney Int Suppl. 2003;63(84):S145-7.

13. Forbes JM, Soldatos G, Thomas MC. Below the radar: advanced glycation end products that detour "around the side". Is $\mathrm{HbA} 1 \mathrm{c}$ not an accurate enough predictor of long term progression and glycaemic control in diabetes? Clin Biochem Rev. 2005;26(4):123-34.

14. Lapolla A, Fedele D, Traldi P. Glyco-oxidation in diabetes and related diseases. Clin Chim Acta. 2005;357(2):236-50.

15. Sharp PS, Rainbow S, Mukhergee S. Serum levels of low molecular weight advanced glycation end products in diabetic subjects. Diabet Med. 2003;20(7):575-9.

16. Rahbar S. The discovery of glycated hemoglobin: a major event in the study of nonenzymatic chemistry in biological systems. Ann N Y Acad Sci. 2005;1043:9-19.

17. Brownlee M. The pathobiology of diabetic complications. A unifying mecanism. Diabetes. 2005;54(6):1615-25.

18. Leslie RD, Beyan H, Sawtell P, Boehm BO, Spector TD, Snieder $\mathrm{H}$. Level of an advanced glycated end product is genetically determined. A study of normal twins. Diabetes. 2003;52(9): 2441-4. 
19. Vlassara H. Advanced glycation in health and disease. Role of the modern environment. Ann N Y Acad Sci. 2005;1043: 452-60.

20. Koschinsky T, He C, Mitsuhashi T, Bucala R, Liu C, Buenting C, et al. Orally absorbed reactive glycation products (glicotoxins): an environmental risk factor in diabetic nephropathy. Proc Natl Acad Sci U S A. 1997;94(12):6476-9.

21. Goldberg T, Cai W, Peppa M, Dardaine V, Baliga BS, Uribarri J, et al. Advanced glycoxidation end products in commonly consumed foods. J Am Diet Assoc. 2004;104(8):1287-91.

22. Vlassara $H$, Palace MR. Diabetes and advanced glycation endproducts. J Intern Med. 2002;251(2):87-101.

23. Cerami C, Founds H, Nicholl I, Mitsuhashi T, Giordano D, Vanapatten $\mathrm{S}$, et al. Tobacco smoke is a source of toxic reactive glycation products. Proc Natl Acad Sci U S A. 1997;94(25): 13915-20.

24. Thornalley PJ. The enzymatic defence against glycation in health, disease and therapeutics: a symposium to examine the concept. Biochem Soc Trans. 2003;6:1341-2.

25. Vlassara H, Palace MR. Glycoxidation: the menace of diabetes and aging. Mt Sinai J Med. 2003;70(4):232-41.

26. Gugliucci $A$, Bendayan M. Renal fate of circulating advanced glycated end products (AGE): evidence for reabsorption and catabolism of AGE-peptides by renal proximal tubular cells. Diabetologia. 1996;39(2):149-60.

27. Nakamura S, Tobita K, Tachikawa T, Akamatsu S, Ohno Y, Kan $A$, et al. Immunohistochemical detection of an AGE, a ligand of macrophage receptor, in peritoneum of CAPD pacients. Kidney Int Suppl. 2003;(84):S152-7.

28. Zheng F, Cai W, Mitsuhashi T, Vlassara H. Lyzosyme enhances renal excretion of advanced glycation endproducts in vivo and suppresses adverse age-mediated cellular effects in vitro: a potential AGE sequestration therapy for diabetic nephropathy? Mol Med. 2001;7(11):737-47.

29. Takeuchi M, Yamagishi S. TAGE (toxics AGEs) hypothesis in various chronic diseases. Med Hypotheses. 2004;63(3):449-52.

30. Poirier O, Nicaud V, Vionnet N, Raoux S, Tarnow L, Vlassara H, et al. Polymorphism screening of four genes encoding advanced glycation end-product putative receptors: association study with nephropathy in type 1 diabetic patients. Diabetes. 2001;50(5):1214-8.

31. Goldin A, Beckman JA, Schimidt AM, Creager MA. Advanced glycation end products. Sparking the development of diabetic vascular injury. Circulation. 2006;114(6):597-605.

32. Lin L. RAGE on the toll road? Cell Mol Immunol. 2006; 3(5):351-8.

33. Yonekura H, Yamamoto Y, Sakurai S, Watanabe T, Yamamoto $H$. Roles of the receptor for advanced glycation endproducts in diabetes-induced vascular injury. J Pharmacol Sci. 2005; 97(3):305-11.

34. Shoji T, Koyama H, Morioka T, Tanaka S, Kizu A, Motoyama K, et al. Receptor for advanced glycation end products is involved in impaired angiogenic response in diabetes. Diabetes. 2006;55(8):2245-55.

35. Myint KM, Yamamoto Y, Doi T, Kato I, Harashima A, Yonekura $\mathrm{H}$, et al. RAGE control of diabetic nephropathy in a mouse model. Effects of RAGE gene disruption and administration of low-molecular weight heparin. Diabetes. 2006; 55(9):2510-22.

36. Basta G, Schimidt AM, De Caterina R. Advanced glycation end products and vascular inflammation: implications for accelerated atherosclerosis in diabetes. Cardiovasc Res. 2004;63(4): 582-92.
37. Antonetti DA, Barber AJ, Bronson SK, Freeman WM, Gardner TW, Jefferson LS, et al. Diabetic retinopathy: seeing beyond glucose-induced microvascular desease. Diabetes. 2006;55(9): 2401-11.

38. Yu DY, Cringle SJ, Su EN, Yu PK, Jerums G, Cooper ME. Pathogenesis and intervention strategies in diabetic retinopathy. Clin Experiment Ophthalmol. 2001;29(3):164-6.

39. Franke S, Dawnczynski J, Strobel J, Niwa T, Stahl P, Stein G. Increased levels of advanced glycation end products in human cataractous lenses. J Cataract Refract Surg. 2003;29(5): 998-1004.

40. Yamamoto Y, Kato I, Doi T, Yonekura H, Ohashi S, Takeuchi M, et al. Development and prevention of diabetic nephropathy in RAGE-overexpressing mice. J Clin Invest. 2001;108(2):261-8.

41. Cooper ME, Gilbert RE, Epstein M. Patophysiology of diabetic nephropathy. Metabolism. 1998;47(12 Suppl 1):3-6.

42. Guven S, Kuenzi JA, Matfin G. Diabetes mellitus. In: Porth CM. Fisiopatologia. $6^{\mathrm{a}}$ ed. Rio de Janeiro: Guanabara Koogan; 2004. p. 896-924.

43. Bierhaus A, Halsbeck M, Humpert PM, Liliensiek B, Dehmer T, Morcos $\mathrm{M}$, et al. Loss of pain perception in diabetes is dependent on a receptor of the imunoglobulin superfamily. J Clin Invest. 2004;114(12):1741-51.

44. Singer A, Clark R. Cutaneous wound healing. N Engl J Med. 1999;341(10):738-46.

45. Monnier VM, Sell DR, Genuth S. Glycation products as markers and predictors of the progression of diabetic complications. Ann N Y Acad Sci. 2005;1043:567-81.

46. Genuth S, Sun W, Cleary P, Sell DR, Dahms W, Malone J, et al. Glycation and carboxymethyllisyne levels in skin collagen predict the risk of future 10-year progression of diabetic retinopathy and nephropathy in the diabetes control and complications trial and epidemiology of diabetes interventions and complications participants with type 1 diabetes. Diabetes. 2005 54(11):3103-11.

47. Meerwaldt R, Links T, Graaff R, Thorpe SR, Baynes JW, Hartog $\mathrm{J}$, et al. Simple noninvasive measurement of skin autofluorescence. Ann N Y Acad Sci. 2005;1043:290-8.

48. Hartog JW, Vries AP, Lutgers HL, Meerwaldt R, Huisman RM, Van Son WJ, et al. Accumulation of advanced glycation end products, measured as skin autofluorescence, in renal disease. Ann N Y Acad Sci. 2005;1043:299-307.

49. Maynard JD, Rohrscheib M, Way JF, Nguyen CM, Ediger MN Noninvasive type 2 diabetes screening: superior sensitivity to fasting plasma glucose and A1C. Diabetes Care. 2007;30(5): 1120-4.

50. McEniery CM. Novel therapeutic strategies for reducing arterial stiffness. Br J Pharmacol. 2006;148(7):881-3.

51. Lin R, Choudhury RP, Cai W, Lu M, Fallon JT, Fisher EA, et al. Dietary glycotoxins promote diabetic atherosclerosis in apolipoprotein E-deficient mice. Atherosclerosis. 2003;168(2): 213-20.

52. Uribarri J, Peppa M, Cai W, Goldberg T, Lu M, He C, et al. Restriction of dietary glicotoxins reduces excessive advanced glycation end products in renal failure patients. J Am Soc Nephrol. 2003;14(3):728-31.

53. Lin R, Reis ED, Dore AT, Lu M, Ghodsi N, Fallon JT, et al. Lowering of dietary advanced glycation endproducts (AGE) reduces neointimal formation after arterial injury in genetically hypercholesterolemic mice. Atherosclerosis. 2002;163(2):303-11.

54. Hofmann S, Dong HJ, Li Z, Cai W, Altomonte J, Thung SN, et al. Improved insulin sensitivity is associated with restricted 
intake of dietary glycoxidation products in $\mathrm{db} / \mathrm{db}$ mouse. Diabetes. 2002;51(7):2082-9.

55. Sandu O, Song K, Cai W, Zheng F, Uribarri J, Vlassara H. Insulin resistance and type 2 diabetes in high-fat-fed mice are linked to high glycotoxin intake. Diabetes. 2005;54(8):2314-9.

56. Peppa M, Brem H, Ehrlich P, Zhang J, Cai W, Li Z, et al. Adverse effects of dietary glycotoxins on wond healing in genetically diabetic mice. Diabetes. 2003;52(11):2805-13.

57. Peppa M, He C, Hattori M, McEvoy R, Zheng F, Vlassara H. Fetal or neonatal low-glycotoxin environment prevents autoimmune diabetes in NOD mice. Diabetes. 2003;52(6):1441-8.

58. Vlassara H, Cai W, Crandall J, Goldberg T, Oberstein R, Dardaine $\mathrm{V}$, et al. Inflamatory mediators are induced by dietary glycotoxins, a major risk factor for diabetic angiopathy. Proc Natl Acad Sci. 2002;99(24):15596-601.

59. Reddy VP, Beyaz A. Inhibitors of the Maillard reaction and AGE breakers as therapeutics for multiple diseases. Drug Discov Today. 2006;11(13-14):646-54.

60. Ahmad MS, Ahmed N. Antiglycation properties of aged garlic extract: possible role in prevention of diabetic complications. J Nutr. 2006;136(3 Suppl):S796-9.

61. Babu PVA, Sabitha KE, Shyamaladevi CS. Green tea impedes dyslipidemia, lipid peroxidation, protein glycation and ameliorates $\mathrm{Ca} 2+-A T P a s e$ and $\mathrm{Na}+/ \mathrm{K}+-$ ATPase activity in the heart of streptozotocin-diabetic rats. Chem Biol Interact. 2006;162(2): 157-64.

62. Lunceford N, Gugliucci A. Ilex paraguariensis extracts inhibit AGE formation more efficiently than green tea. Fitoterapia. 2005;76(5):419-27.

63. Pari L, Venkateswaran S. Effect of an aqueous extract of Phaseolus vulgaris on the properties of tail tendon collagen of rats with streptozotocin-induced diabetes. Braz J Med Biol Res. 2003;36(7):861-70.
64. Mustata GT, Rosca M, Biemel KM, Reihl O, Smith MA, Viswanathan $A$, et al. Paradoxical effects of green tea (camellia sinensis) and antioxidant vitamins in diabetic rats. Diabetes. 2005;54(2):517-26.

65. Osawa T, Kato Y. Protective role of antioxidative food factors in oxidative stress caused by hyperglycemia. Ann N Y Acad Sci. 2005;1043:440-51

66. Babaei-Jadidi R, Karachalias N, Ahmed N, Battah S, Thornalley PJ. Prevention of incipient diabetic nephropathy by highdose thiamine and benfotiamine. Diabetes. 2003;52(8): 2110-20.

67. Stirban A, Negrean M, Stratmann B, Gawlowski T, Horstmann $\mathrm{T}$, Gotting $\mathrm{C}$, et al. Benfotiamine prevents macro- and microvascular endothelial dysfunction and oxidative stress following a meal rich in advanced glycation end products in individuals with type 2 diabetes. Diabetes Care. 2006;29(9): 2064-71.

68. McCarty MF. The low-AGE content of low-fat vegan diets could benefit diabetics - though concurrent taurine supplementation may be needed to minimize endogenous AGE production. Med Hypotheses. 2005;64(2):394-8.

69. Hobart LJ, Seibel I, Yeargans GS, Seidler NW. Anti-crosslinking properties of carnosine: significance of histidine. Life Sci. 2004;75(11):1379-89.

Endereço para correspondência:

Júnia Helena Porto Barbosa

Av. José Sampaio Luz, 1271, apto. 101

57035-260 Maceió-AL

E-mail: juniap@gmail.com 